\title{
WEAK COMPACTNESS IN THE DUAL SPACE OF A JB*-TRIPLE IS COMMUTATIVELY DETERMINED
}

\author{
FRANCISCO J. FERNÁNDEZ-POLO and ANTONIO M. PERALTA*
}

\begin{abstract}
We prove the following criterium of weak compactness in the dual of a JB*-triple: a bounded set $K$ in the dual of a $\mathrm{JB}^{*}$-triple $E$ is not relatively weakly compact if and only if there exist a sequence of pairwise orthogonal elements $\left(a_{n}\right)$ in the closed unit ball of $E$, a sequence $\left(\varphi_{n}\right)$ in $K$, and $\vartheta>0$ satisfying that $\left|\varphi_{n}\left(a_{n}\right)\right|>\vartheta$ for all $n \in \mathrm{N}$. This solves a question stimulated by the main result in [11] and posed in [9].
\end{abstract}

\section{Introduction and Preliminaries}

Relatively weakly compact subsets in the dual of a $\mathrm{C}^{*}$-algebra have been intensively studied during the last fifty years. The first precedent appears in a paper by A. Grothendieck in 1953 (see [15]). This forerunner establishes the following characterization of weak compactness in the dual of a $C(\Omega)$-space: a bounded subset $K \subseteq C(\Omega)^{*}$ is not relatively weakly compact if and only if there exists a sequence $\left(O_{n}\right)$ of pairwise disjoint open subsets of $\Omega$ such that $\lim _{n \rightarrow \infty} \sup \left\{\left|\mu\left(O_{n}\right)\right|: \mu \in K\right\} \neq 0$. Urysohn's lemma allows us to replace the $O_{n}$ 's by norm-one positive continuous functions on $\Omega$ with mutually disjoint supports.

When $K$ is a bounded set in the predual of a von Neumann algebra $M$, M. Takesaki [26] and C. Akemann [1] (see also [27, Theorem III.5.4]) proved that $K$ is not relatively weakly compact if and only if there exists a sequence $\left(p_{n}\right)$ of pairwise orthogonal projections in $M$ such that $\lim _{n \rightarrow \infty} \sup \left\{\left|\phi\left(p_{n}\right)\right|\right.$ : $\phi \in K\} \neq 0$. That is, weak compactness in $M_{*}$ is determined by the abelian subalgebras of $M$. Consequently, relatively weakly compact subsets in the dual of a $\mathrm{C}^{*}$-algebra $A$ are commutatively determined by the abelian subalgebras of $A^{* *}$.

In [24] H. Pfitzner showed that weak compactness in the dual of a $\mathrm{C}^{*}$ algebra $A$ is in fact determined by the abelian subalgebras of $A$. Concretely, a bounded set $K \subseteq A^{*}$ fails to be relatively weakly compact if and only if there

\footnotetext{
* Authors partially supported by M.I.C. project no. MTM2008-02186, and Junta de Andalucía grants FQM0199 and FQM1215.

Received June 9, 2008; in final form October 15, 2008.
} 
exist a positive $\theta$, a sequence $\left(a_{n}\right)$ of pairwise orthogonal positive elements in the closed unit ball of $A$ and a sequence $\left(\varphi_{n}\right)$ in $K$ satisfying $\left|\varphi_{n}\left(a_{n}\right)\right|>\theta$, for every $n \in \mathrm{N}$ (compare [12] for a new and shorter proof).

$\mathrm{C}^{*}$-algebras belong to a more general class of complex Banach spaces in which the geometric, holomorphic, and algebraic structure mutually interplay. We are referring to the class of $\mathrm{JB}^{*}$-triples. We recall (see [21]) that a $J B^{*}$ triple is a complex Banach space $E$ equipped with a continuous triple product $\{\cdot, \cdot, \cdot\}: E \times E \times E \rightarrow E$, which is symmetric and linear in the first and third variables, conjugate linear in the second variable and satisfies:

(i) (Jordan Identity) $L(a, b) L(x, y)=L(x, y) L(a, b)+L(L(a, b) x, y)-$ $L(x, L(b, a) y)$, where $L(a, b)$ is the operator on $E$ given by $L(a, b) x=$ $\{a, b, x\}$

(ii) $L(a, a)$ is a hermitian operator with non-negative spectrum;

(iii) $\|L(a, a)\|=\|a\|^{2}$.

Every $\mathrm{C}^{*}$-algebra is a $\mathrm{JB}^{*}$-triple with respect to the product $\{x, y, z\}=\frac{1}{2}\left(x y^{*} z\right.$ $\left.+z y^{*} x\right)$, and every $\mathrm{JB}^{*}$-algebra is a $\mathrm{JB}^{*}$-triple under the triple product $\{x, y, z\}=\left(x \circ y^{*}\right) \circ z+\left(z \circ y^{*}\right) \circ x-(x \circ z) \circ y^{*}$.

A $\mathrm{JBW}^{*}$-triple is a $\mathrm{JB}^{*}$-triple which is also a dual Banach space (with a unique isometric predual [3]). It is known that the second dual of a JB*-triple is a $\mathrm{JBW}^{*}$-triple (compare [8]). Further, the triple product of every $\mathrm{JBW}^{*}$-triple is separately weak*-continuous [3].

The above quoted results of Takesaki and Akemann were extended in [23] to characterize relatively weakly compact subsets in the predual of a JBW*-triple.

A $J C^{*}$-triple is a norm-closed subspace of a $\mathrm{C}^{*}$-algebra which is closed under the ternary product $\{x, y, z\}=\frac{1}{2}\left(x y^{*} z+z y^{*} x\right)$. $\mathrm{JC}^{*}$-triples form an intermediate class of complex Banach spaces between $\mathrm{C}^{*}$-algebras and $\mathrm{JB}^{*}$ triples. A criterium for weak compactness in the dual of a $\mathrm{JC}^{*}$-triple, which is also a generalization of Pfitzner's result, was established in [11]. This criterium assures that a bounded subset in the dual space of a $\mathrm{JC}^{*}$-triple $E$ is relatively weakly compact if and only if its restriction to any abelian maximal subtriple $C$ of $E$ is relatively weakly compact in the dual of $C$. However, as pointed out by C. M. Edwards in [9], "whether the results hold for general JB*-triples remains an open question". The main result of this paper gives a positive answer to this question for general JB*-triples (see Theorem 2.3). The solution presented in this paper is itself a novelty which simplifies the results in [11] with a new and shorter orthogonalization process based on Bergmann operators.

Reference [6] is a basic forerunner of the problem studied in this paper. Briefly speaking, we could say [6] contains a partial answer for our problem in terms of Pelczynski's Property (V). We recall that a series $\sum_{n \geq 1} z_{n}$ in a Banach space $X$ is called weakly unconditionally convergent (w.u.c. for short) 
if for each $\varphi \in X^{*}$ we have $\sum_{n=1}^{\infty}\left|\varphi\left(z_{n}\right)\right|<\infty$, equivalently, there exists $C>0$ such that for any finite subset $\mathscr{F} \subset \mathrm{N}$ and $\left|\varepsilon_{k}\right|=1$ in $\mathrm{C}$ we have $\left\|\sum_{k \in \mathscr{F}} \varepsilon_{k} z_{k}\right\| \leq C$, (see, for example, [7, Theorem 6 in Chapter 5]). It is clear that every bounded linear operator between Banach spaces preserves w.u.c. series. A Banach space $X$ has property $(V)$ if for any (bounded) non relatively weakly compact set $K \subseteq X^{*}$ there exists a w.u.c. series $\sum_{n} x_{n}$ in $X$ such that $\sup _{\varphi \in X^{*}}\left|\varphi\left(x_{n}\right)\right|$ does not converge to zero. It is established in [6] that every $\mathrm{JB}^{*}$-triple satisfies property $(V)$. We shall see later that every bounded sequence of mutually orthogonal elements in a $\mathrm{JB}^{*}$-triple defines a w.u.c. series, however the reciprocal statement need not hold in general. We shall establish a new orthogonalization method to construct sequences of mutually orthogonal elements from w.u.c. series.

\subsection{Preliminaries}

Let $X$ and $Y$ be two Banach spaces, throughout the paper, the symbol $L(X, Y)$ will stand for the space of all bounded linear operators from $X$ to $Y$. We shall write $L(X)$ for the space $L(X, X)$.

$\mathrm{A} \mathrm{JB}^{*}$-triple $E$ is said to be abelian if $\{\{x, y, z\}, u, v\}=\{x, y,\{z, u, v\}\}=$ $\{x,\{y, z, u\}, v\}$, for all $x, y, z, u, v \in E$. The $\mathrm{JB}^{*}$-subtriple generated by a single element is always abelian.

Let $x$ be an element in a JB*-triple $E$. Throughout the paper the symbol $E_{x}$ will denote the norm-closed subtriple of $E$ generated by $x$. It is known that $E_{x}$ is $\mathrm{JB}^{*}$-triple isomorphic to the $\mathrm{C}^{*}$-algebra $C_{0}(L)$ of all complex-valued continuous functions on $L$ vanishing at 0 , where $L$ is a locally compact subset of $(0,\|x\|]$ satisfying that $L \cup\{0\}$ is compact. Further, there exists a JB*-triple isomorphism $\Psi: E_{x} \rightarrow C_{0}(L)$ which satisfies $\Psi(x)(t)=t$, for all $t$ in $L$ (compare [20,4.8] and [21, 1.15]). In particular, given a natural $n$, the symbol $x^{\frac{1}{2 n-1}}$ makes sense as an element of $E_{x} \cong C_{0}(L)$.

An element $u$ in a $\mathrm{JB}^{*}$-triple $E$ is said to be a tripotent if $u=\{u, u, u\}$. Given a tripotent $u \in E$, the mappings $P_{i}(u): E \rightarrow E_{i},(i=0,1,2)$, defined by

$$
\begin{aligned}
& P_{2}(u)=L(u, u)\left(2 L(u, u)-\mathrm{id}_{E}\right), \\
& P_{1}(u)=4 L(u, u)\left(\operatorname{id}_{E}-L(u, u)\right), \quad \text { and } \\
& P_{0}(u)=\left(\operatorname{id}_{E}-L(u, u)\right)\left(\operatorname{id}_{E}-2 L(u, u)\right),
\end{aligned}
$$

are contractive linear operators. For each $j=0,1,2, P_{j}(u)$ is the projection onto the eigenspace $E_{j}(u)$ of $L(u, u)$ corresponding to the eigenvalue $\frac{j}{2}$ and

$$
E=E_{2}(u) \oplus E_{1}(u) \oplus E_{0}(u)
$$

is the Peirce decomposition of $E$ relative to $u$. Furthermore, the following 
Peirce rules are satisfied,

$$
\begin{aligned}
\left\{E_{2}(u), E_{0}(u), E\right\} & =\left\{E_{0}(u), E_{2}(u), E\right\}=0, \\
\left\{E_{i}(u), E_{j}(u), E_{k}(u)\right\} & \subseteq E_{i-j+k}(u),
\end{aligned}
$$

where $E_{i-j+k}(u)=0$ whenever $i-j+k \notin\{0,1,2\}$ (compare [13]).

When $W$ is a $\mathrm{JBW}^{*}$-triple, the $\mathrm{JBW}^{*}$-subtriple generated by a norm-one element $x \in W$ coincides with the weak*-closure, $\overline{W_{x}}{ }^{*}$, of $W_{x}$. By [18, Lemma 3.11] there exists a $\mathrm{JBW}^{*}$-triple isomorphism, $\Psi$, between ${\overline{W_{x}}}^{{ }^{*}}$ and a commutative $\mathrm{W}^{*}$-algebra $C$. We shall write $r(x)=\Psi^{-1}(1)$, where 1 denotes the unit element in $C$. It is clear that $r(x)$, commonly termed the range tripotent of $x$, is a tripotent in $W$. Moreover, $r(x)$ coincides with the weak*-limit of the sequence $x^{\frac{1}{2 n-1}},(n \in \mathrm{N})$. It is also known that the $\mathrm{JBW}^{*}$-algebra $E_{2}^{* *}(r(x))$ contains $x$ as a positive element (compare [10]).

Given a $\mathrm{JBW}^{*}$-triple $W$, a norm-one element $\varphi$ in $W_{*}$ and a norm-one element $z$ in $W$ with $\varphi(z)=1$, it follows from [2, Proposition 1.2] that the assignment

$$
(x, y) \mapsto \varphi\{x, y, z\}
$$

defines a positive sesquilinear form on $W$. Further, for every norm-one element $w$ in $W$ satisfying $\varphi(w)=1$, we have $\varphi\{x, y, z\}=\varphi\{x, y, w\}$, for all $x, y \in W$. The mapping $x \mapsto\|x\|_{\varphi}:=(\varphi\{x, x, z\})^{\frac{1}{2}}$, defines a prehilbertian seminorm on $W$. The Strong*-topology (noted by $S^{*}\left(W, W_{*}\right)$ ) is the topology on $W$ generated by the family $\left\{\|\cdot\|_{\varphi}: \varphi \in W_{*},\|\varphi\|=1\right\}$. This topology was introduced by T. J. Barton and Y. Friedman in [2].

When $\varphi$ is an element in the dual of a JB*-triple $E$, the prehilbertian seminorm $\|\cdot\|_{\varphi}$ is defined on $E^{* *}$ (and hence on $E$ ) by the assignment

$$
x \mapsto\|x\|_{\varphi}:=(\varphi\{x, x, z\})^{\frac{1}{2}},
$$

where $z$ is a norm-one element in $E^{* *}$ with $\varphi(z)=\|\varphi\|$. The inequality

$$
\|\{x, y, z\}\| \leq\|x\|\|y\|\|z\|
$$

holds for every $x, y$ and $z$ in a $\mathrm{JB}^{*}$-triple $E$ (compare [14, Corollary 3]). Consequently,

$$
\|x\|_{\varphi} \leq\|\varphi\|^{\frac{1}{2}}\|x\|,
$$

for all $\varphi \in E^{*}$ and $x \in E$.

For each element $a$ in a JB*-triple $E$, the conjugate linear mapping $Q(a)$ from $E$ to itself is defined, for each element $b$ in $E$, by $Q(a)(b):=\{a, b, a\}$. Let $x, y$ be two elements in $E$. The Bergmann operator $B(x, y): E \rightarrow E$ 
is defined by $B(x, y)(z)=z-2 L(x, y)(z)+Q(x) Q(y)(z)$, for all $z$ in $E$ (compare [22] or [28, page 305]). In the particular case of $u$ being a tripotent, it is known that $P_{0}(u)=B(u, u)$.

Let $x$ be a symmetric element in a unital $\mathrm{JB}^{*}$-algebra $A$. The operator $U_{x}: A \rightarrow A$ is defined by $U_{x}(y):=2(y \circ x) \circ x-x^{2} \circ y$, for all $y$ in $A$. When $A$ is regarded as a JB*-triple, we have $U_{x}(y)=Q(x)\left(y^{*}\right), \forall y \in A$. Since by [16, Lemma 2.4.21] $U_{x}^{2}=U_{x^{2}}$, we deduce that

$$
Q(x)^{2}(y)=U_{x}^{2}(y)=U_{x^{2}}(y)=Q\left(x^{2}\right)\left(y^{*}\right), \quad \forall y \in A .
$$

We also have $2 L(x, x)(y)=2\left(x^{2} \circ y+(y \circ x) \circ x-(y \circ x) \circ x\right)=2 x^{2} \circ y$, for all $y \in A$. Therefore, for each $y \in A$ we have

$$
B(x, x)(y)=y-2 L(x, x)(y)+Q(x)^{2}(y)=Q\left(1-x^{2}\right)\left(y^{*}\right),
$$

which implies that $\|B(x, x)\| \leq 1$, whenever $x$ belongs to the closed unit ball of $A$.

A tripotent $u$, in a $\mathrm{JB}^{*}$-triple $E$, is said to be bounded if there exists a normone element $x \in E$ such that $L(u, u) x=u$. The element $x$ is a bound of $u$ and in this case we write $u \leq x$. We shall write $y \leq u$ whenever $y$ is a positive element in the $\mathrm{JB}^{*}$-algebra $E_{2}(u)$ (compare [11, pages 79-80]).

Lemma 1.1. Let $x$ be a symmetric element in the closed unit ball of a $J B^{*}$-algebra $A$. Then $B(x, x)$ is a contractive operator. Moreover, if $p$ is a projection in $A$ with $p \leq x$, then $B(x, x)(y)$ belongs to $A_{0}(p)$, for every $y$ in A.

Proof. We may assume that $A$ is unital. The comments preceding this lemma guarantee that $\|B(x, x)\| \leq 1$ and $B(x, x) y=Q\left(1-x^{2}\right)\left(y^{*}\right),(y \in A)$. Since $p \leq x^{2} \leq 1$, we have $0 \leq 1-x^{2} \leq 1-p$, and hence $1-x^{2}$ belongs to $A_{0}(p)$. Finally, it follows, by Peirce rules, that $B(x, x) y \in A_{0}(p)$.

Lemma 1.1 above can be now extended to JB*-triples.

Lemma 1.2. Let $E$ be a $J B^{*}$-triple, e a tripotent in $E$, and $x$ a normone element in $E$ with $e \leq x$. Then $B(x, x)$ is a contractive operator and $B(x, x)(y)$ belongs to $E_{0}(e)$, for every $y$ in $E$.

Proof. By [14, Corollary 1] we may suppose that $E$ embeds as a subtriple into a JBW*-algebra, $A$, of the form $L(H) \bigoplus^{\infty} N$, where $H$ is a complex Hilbert space and $N$ is an $\ell_{\infty}$-sum of finite-dimensional simple $\mathrm{JB}^{*}$-algebras.

We may then assume that

$$
e \leq x(\leq r(x))
$$


in the $\mathrm{JBW}^{*}$-algebra $A$, where $r(x)$ is the range tripotent of $x$ in $A$. From [4, Lemma 2.3] and [22, Corollary 5.12] there exists a weak*-continuous isometric triple embedding $T$ from $A$ into $A$, such that $T(r(x)$ ) (and hence $T(e)$ ) is a projection in $A$. It is easy to check that $0 \leq T(e) \leq T(x) \leq T(r(x))$. By Lemma 1.1, we have $T(B(x, x)(y))=B(T(x), T(x))(T(y)) \in A_{0}(T(e))$, for every $T(y) \in T(E) \subseteq A$. Therefore, $B(x, x)(y) \in A_{0}(e) \cap E=E_{0}(e)$, for all $y \in E$.

Another central notion in the paper is the concept of orthogonality. Two elements $a, b$ in a $\mathrm{JB}^{*}$-triple, $E$, are said to be orthogonal (written $a \perp b$ ) if $L(a, b)=0$. Lemma 1 in [5] shows that $a \perp b$ if and only if one of the following statements holds:

$$
\begin{gathered}
\{a, a, b\}=0 ; \quad a \perp r(b) ; \quad r(a) \perp r(b) ; \quad E_{2}^{* *}(r(a)) \perp E_{2}^{* *}(r(b)) ; \\
r(a) \in E_{0}^{* *}(r(b)) ; \quad a \in E_{0}^{* *}(r(b)) ; \quad b \in E_{0}^{* *}(r(a)) ; \quad E_{a} \perp E_{b} .
\end{gathered}
$$

The Peirce rule (1) shows that for each tripotent $u$ in a JB*-triple $E, E_{0}(u) \perp$ $E_{2}(u)$. The Jordan identity and the above reformulations assure that

$$
a \perp\{x, y, z\}, \quad \text { whenever } a \perp x, y, z .
$$

Let $A$ be a $C^{*}$-algebra. Two elements $a, b \in A$ are said to be orthogonal for the $\mathrm{C}^{*}$-algebra product if $a b^{*}=b^{*} a=0$. However, $A$ also enjoys a structure of $\mathrm{JB}^{*}$-triple. We have, a priory, two notions of orthogonality in $A$. It can be checked, from the above reformulations, that two elements $a, b$ in $A$ are orthogonal for the $\mathrm{C}^{*}$-algebra product if and only if they are orthogonal when $A$ is considered as a $\mathrm{JB}^{*}$-triple.

For every tripotent $e$ in a $\mathrm{JB}^{*}$-triple $E$, the formula

$$
\left\|P_{2}(e)(x)+P_{0}(e)(x)\right\|=\max \left\{\left\|P_{2}(e)(x)\right\|,\left\|P_{0}(e)(x)\right\|\right\},
$$

holds for every $x$ in $E$ (compare [13, Lemma 1.3]). In particular, if $\left\{x_{1}, \ldots, x_{m}\right\}$ is a set of mutually orthogonal elements in a $\mathrm{JB}^{*}$-triple $E$, it follows from the above equivalent reformulations of orthogonality and the previous formula, that the $\mathrm{JB}^{*}$-subtriple generated by the set $\left\{x_{1}, \ldots, x_{m}\right\}$ coincides with the $\ell_{\infty}$-sum $\bigoplus_{k=1, \ldots, m}^{\infty} E_{x_{k}}$ and hence it is $\mathrm{JB}^{*}$-triple isomorphic to an abelian $\mathrm{C}^{*}$-algebra.

We deduce from the above paragraph that every bounded sequence of pairwise orthogonal elements in a JB*-triple defines a w.u.c. series. 


\section{Main result}

The aim of this section is to prove that weak compactness in the dual of a $\mathrm{JB}^{*}$-triple is commutatively determined. Bergmann operators, wisely used, turn to be a powerful tool in orthogonalization processes. More concretely, we shall make use of appropriated Bergmann operators to orthogonalize weakly unconditional convergent series in $\mathrm{JB}^{*}$-triples.

Lemma 2.1. Let $E$ be a JB-triple, $v$ a tripotent in $E$, and $\varphi$ an element in the closed unit ball of $E^{*}$. Then for each $y \in E_{2}(v)$ with $\|y\| \leq 1$ we have

$$
|\varphi(x-B(y, y)(x))|<21\|x\|\|v\|_{\varphi},
$$

for every $x \in E$.

Proof. By Peirce rules we have $L(y, y)(x) \in E_{2}(v) \oplus E_{1}(v)$ and $Q(y)^{2}(x)$ $\in E_{2}(v)$. Since $x-B(y, y)(x)=2 L(y, y)(x)-Q(y)^{2}(x)$, the desired statement follows from [11, Lemma 3.2].

We shall also need the following strengthening version of [11, Lemma 3.4].

Lemma 2.2. Let $E$ be a JB*-triple, $\theta>0, \delta_{n}>0(n \in \mathrm{N})$, and let $\left\{\varphi_{1}\right\} \cup\left\{\varphi_{n}\right\}_{n \geq 2}$ be a sequence of functionals in the closed unit ball of $E^{*}$. Given an element $x$ in the closed unit ball of $E$, satisfying $\left|\varphi_{1}(x)\right|>\theta$ and $\|x\|_{\varphi_{n}}<\delta_{n}, n \geq 2$, there exist two elements $a, y$ in the unit ball of $E_{x}$, and two tripotents $u, v$ in $\left(E_{x}\right)^{* *}$ such that $a \leq u \leq y \leq v,\left|\varphi_{1}(a)\right|>\frac{3}{4} \theta$, and $\|v\|_{\varphi_{n}}<\frac{8}{\theta} \delta_{n}, n \geq 2$.

Proof. We have already commented that $E_{x}$ is $\mathrm{JB}^{*}$-triple isomorphic to the $\mathrm{C}^{*}$-algebra $C_{0}(L)$, where $L$ is a locally compact subset of $(0,\|x\|]$ satisfying that $L \cup\{0\}$ is compact. Moreover, there exists a $\mathrm{JB}^{*}$-triple isomorphism $\Psi: E_{x} \rightarrow C_{0}(L)$ satisfying $\Psi(x)(t)=t$, for all $t$ in $L$. By slight abuse of notation, $E_{x}$ and $C_{0}(L)$ will be identified.

Let $a, y \in C_{0}(L)$ be the functions defined by

$$
\begin{aligned}
& a(t):=\left\{\begin{array}{ll}
0, & \text { if } 0 \leq t \leq \frac{\theta}{4} \\
2 t-\frac{\theta}{2}, & \text { if } \frac{\theta}{4} \leq t \leq \frac{\theta}{2} \\
t, & \text { if } \frac{\theta}{2} \leq t \leq\|x\|
\end{array} ;\right. \\
& y(t):= \begin{cases}0, & \text { if } 0 \leq t \leq \frac{\theta}{8} \\
\frac{8}{\theta}\left(t-\frac{\theta}{8}\right), & \text { if } \frac{\theta}{8} \leq t \leq \frac{\theta}{4} \\
1, & \text { if } \frac{\theta}{4} \leq t \leq\|x\| .\end{cases}
\end{aligned}
$$


Since $\|x-a\|<\frac{\theta}{4}$ and $\left|\varphi_{1}(x)\right|>\theta$ it follows that $\left|\varphi_{1}(a)\right|>\frac{3}{4} \theta$.

The element $x$ decomposes as the sum of two orthogonal elements $x=$ $x \chi_{\left[\frac{\theta}{8},\|x\|\right]}+x \chi_{\left[0, \frac{\theta}{8}\right)}\left(\right.$ in $\left.\left(E_{x}\right)^{* *}\right)$. Since $\|\cdot\|_{\varphi_{n}}^{2}$ is additive when applied to the sum of orthogonal elements, we get $\left\|x \chi_{\left[\frac{\theta}{8},\|x\|\right]}\right\|_{\varphi_{n}}<\delta_{n}$. We define $u=\chi_{\left[\frac{\theta}{4},\|x\|\right]}$, $v=\chi_{\left[\frac{\theta}{8},\|x\|\right]}\left(\right.$ in $\left.\left(E_{x}\right)^{* *}\right)$, which clearly satisfy that $a \leq u \leq y \leq v$.

Since $\|\cdot\|_{\varphi}$ is an order-preserving map on the set of positive elements in $\left(E_{x}\right)^{* *}\left(\left[11\right.\right.$, Lemma 3.3]), we have that $\|v\|_{\varphi_{n}} \leq\left\|\frac{8}{\theta} x \chi_{\left[\frac{\theta}{8},\|x\|\right]}\right\|_{\varphi_{n}}<\frac{8}{\theta} \delta_{n}$ $(n \geq 2)$, which finishes the proof.

Our main result can be stated now.

Theorem 2.3. Let $E$ be a JB*-triple and $K$ be a bounded subset in $E^{*}$. The following are equivalent:

a) $K$ is not relatively weakly compact.

b) There exist a sequence of pairwise orthogonal elements $\left(a_{n}\right)$ in the closed unit ball of $E$, a sequence $\left(\varphi_{n}\right)$ in $K$, and $\vartheta>0$ satisfying that $\left|\varphi_{n}\left(a_{n}\right)\right|>$ $\vartheta$ for all $n \in \mathrm{N}$.

$\left.\mathrm{b}^{\prime}\right)$ There exists a subtriple $\mathscr{C}$ of $E$ isometric to an abelian $C^{*}$-algebra such that the restriction of $K$ to it is not relatively weakly compact.

Proof. a) $\Rightarrow$ b). Since JB*-triples have Pelczynski's Property (V) (compare [6]) there exist $\theta>0,\left(\varphi_{n}\right) \subset K$ and a w.u.c. series $\sum_{n \geq 1} z_{n}$ in $E$ with $\left\|z_{n}\right\| \leq 1$, such that $\left|\varphi_{n}\left(z_{n}\right)\right|>\theta, \forall n \in \mathrm{N}$. We may assume that $K$ is contained in the closed unit ball of $E^{*}$.

Let us fix a decreasing sequence $\left(\delta_{n}\right)$ of positive numbers satisfying $\frac{336}{\theta} \sum_{n=1}^{\infty} \delta_{n}<\frac{\theta}{2}$. We shall construct, inductively, a sequence $\left(a_{n}\right)$ of mutually orthogonal elements in the closed unit ball of $E$, infinite subsets $\mathrm{N} \supsetneq$ $N_{1} \supsetneq N_{2} \supsetneq \cdots \supsetneq N_{n-1} \supsetneq N_{n} \supsetneq \cdots$ and a strictly increasing mapping $\sigma: \mathrm{N} \rightarrow \mathrm{N}$ such that for each natural $n$ there exists a w.u.c. series $\sum_{k \in N_{n}} z_{n, k}$ in $E$ with $\left\|z_{n, k}\right\| \leq 1$,

$$
\begin{aligned}
& z_{n, k} \perp a_{j}, \quad \text { for all } \quad j \in\{1, \ldots, n\}, \quad k \in N_{n}, \\
& \left|\varphi_{\sigma(i)}\left(a_{i}\right)\right|>\frac{3}{8} \theta, \quad i=1, \ldots, n, \\
& \left|\varphi_{k}\left(z_{n, k}\right)\right|>\theta-\frac{336}{\theta} \sum_{j=1}^{n} \delta_{j}>\frac{\theta}{2}, \quad k \in N_{n} .
\end{aligned}
$$

and

To define $a_{1}$, choose $j_{1} \in \mathrm{N}$ with $\frac{1}{j_{1}}<\frac{1}{C^{2}} \delta_{1}^{2}$, where $C$ is the positive constant associated to the w.u.c. series $\sum_{n \geq 1} z_{n}$ (see comments in the Introduction). 
Since every Hilbert space is of cotype 2 (compare [25, page 32]) we have

$$
\begin{aligned}
& \frac{1}{j_{1}} \sum_{k=1}^{j_{1}}\left\|z_{k}\right\|_{\varphi_{m}}^{2} \leq \frac{1}{j_{1}} \int_{D}\left\|\sum_{k=1}^{j_{1}} \varepsilon_{k} z_{k}\right\|_{\varphi_{m}}^{2} d \mu \\
& \leq \frac{1}{j_{1}} \int_{D}\left\|\varphi_{m}\right\|\left\|\sum_{k=1}^{j_{1}} \varepsilon_{k} z_{k}\right\|^{2} d \mu \leq \frac{C^{2}}{j_{1}}<\delta_{1}^{2},
\end{aligned}
$$

where $m \in \mathrm{N}, D=\{-1,1\}^{\mathrm{N}}, \varepsilon_{k} \in\{ \pm 1\}$ and $\mu$ is the uniform probability measure on $D$. Since the above inequality is satisfied for every $m \in \mathbb{N}$, there exist $\sigma(1) \in\left\{1, \ldots, j_{1}\right\}$ and an infinite subset $N_{1} \subset \mathrm{N}$ such that $\sigma(1)<$ $\min N_{1}$ and $\left\|z_{\sigma(1)}\right\|_{\varphi_{m}}<\delta_{1}$, for every $m \in N_{1}$.

Applying Lemma 2.2 to $z_{\sigma(1)}$ and $\left\{\varphi_{\sigma(1)}\right\} \cup\left\{\varphi_{m}\right\}_{m \in N_{1}}$ we obtain two elements $a_{1}, y_{1}$ in the closed unit ball of $E_{z_{\sigma(1)}}$ and two tripotents $u_{1}, v_{1} \in E^{* *}$ such that $a_{1} \leq u_{1} \leq y_{1} \leq v_{1}$,

$$
\left|\varphi_{\sigma(1)}\left(a_{1}\right)\right|>\frac{3}{4} \theta>\frac{3}{8} \theta, \quad \text { and } \quad\left\|v_{1}\right\|_{\varphi_{m}}<\frac{8}{\theta} \delta_{1}<\frac{16}{\theta} \delta_{1}, \quad m \in N_{1} .
$$

We define $z_{1, k}:=B\left(y_{1}, y_{1}\right) z_{k},\left(k \in N_{1}\right)$, which are elements in the closed unit ball of $E$ by Lemma 1.2. Clearly $\sum_{k \in N_{1}} z_{1, k}$ also is a w.u.c. series. Lemma 1.2 assures that $z_{1, k}$ is contained in $E \cap E_{0}^{* *}\left(u_{1}\right)$. Since $a_{1} \in E_{2}^{* *}\left(u_{1}\right)$, we deduce that $a_{1} \perp z_{1, k}, \forall k \in \mathrm{N}$ (compare with the reformulations of orthogonality given in page 312). Moreover $\left\|\sum_{k \in \mathscr{F}} \varepsilon_{k} z_{1, k}\right\|=\left\|B\left(y_{1}, y_{1}\right)\left(\sum_{k \in \mathscr{F}} \varepsilon_{k} z_{k}\right)\right\| \leq C$, for every finite $\mathscr{F} \in N_{1}$ and $\left|\varepsilon_{k}\right|$ in C. Now, noticing that $y_{1} \in E_{2}^{* *}\left(v_{1}\right)$, Lemma 2.1 applies to assure that

$$
\left|\varphi_{k}\left(z_{1, k}\right)\right| \geq\left|\varphi_{k}\left(z_{k}\right)\right|-\left|\varphi_{k}\left(z_{k}-z_{1, k}\right)\right|>\theta-21 \frac{16}{\theta} \delta_{1}\left(>\frac{\theta}{2}\right),
$$

for all $k \in N_{1}$.

Suppose now, in our inductive step, that $a_{1}, \ldots, a_{n}, N_{n} \subsetneq N_{n-1} \subsetneq \cdots \subsetneq$ $N_{1} \subsetneq \mathrm{N}, \sigma(1)<\sigma(2)<\cdots<\sigma(n)$, and the w.u.c. series $\sum_{k \in N_{n}} z_{n, k}$ in $E$ have been constructed satisfying the corresponding induction hypothesis.

Take $j_{n+1} \in \mathrm{N}$ with $\frac{1}{j_{n+1}}<\frac{1}{C^{2}} \delta_{n+1}^{2}$ and a subset $D_{n+1} \subset N_{n}$ with exactly $j_{n+1}$ elements. As before, for $m \in N_{n}$ we have

$$
\begin{aligned}
\frac{1}{j_{n+1}} \sum_{k \in D_{n+1}}\left\|z_{n, k}\right\|_{\varphi_{m}}^{2} \leq & \frac{1}{j_{n+1}} \int_{D}\left\|\sum_{k \in D_{n+1}} \varepsilon_{k} z_{n, k}\right\|_{\varphi_{m}}^{2} d \mu \\
& \leq \frac{1}{j_{n+1}} \int_{D}\left\|\varphi_{m}\right\|\left\|\sum_{k \in D_{n+1}} \varepsilon_{k} z_{n, k}\right\|^{2} d \mu \leq \frac{C^{2}}{j_{n+1}}<\delta_{n+1}^{2},
\end{aligned}
$$


hence there exist $\sigma(n+1) \in D_{n+1}$ and an infinite subset $N_{n+1} \subseteq N_{n}$ such that $\sigma(n+1)<\min N_{n+1}$ and $\left\|z_{n, \sigma(n+1)}\right\|_{\varphi_{m}}<\delta_{n+1}$, for every $m \in N_{n+1}$.

Applying Lemma 2.2 to $z_{n, \sigma(n+1)}$ and $\left\{\varphi_{\sigma(n+1)}\right\} \cup\left\{\varphi_{m}\right\}_{m \in N_{n+1}}$ we obtain two elements $a_{n+1}, y_{n+1}$ in the closed unit ball of $E_{z_{n, \sigma(n+1)}}$ and two tripotents $u_{n+1}, v_{n+1} \in\left(E_{z_{n, \sigma(n+1)}}\right)^{* *}$ such that $a_{n+1} \leq u_{n+1} \leq y_{n+1} \leq v_{n+1}$,

$$
\left|\varphi_{\sigma(n+1)}\left(a_{n+1}\right)\right|>\frac{3}{8} \theta, \quad \text { and } \quad\left\|v_{n+1}\right\|_{\varphi_{m}}<\frac{16}{\theta} \delta_{n+1}, \quad m \in N_{n+1} .
$$

By the induction hypothesis, $z_{n, k} \perp a_{j}$, for all $j \in\{1, \ldots, n\}, k \in N_{n}$. Since $a_{n+1}, y_{n+1}, u_{n+1}$, and $v_{n+1}$ belong to $\left(E_{z_{n, \sigma(n+1)}}\right)^{* *}$, the equivalent reformulations of orthogonality given in page 312 , guarantee that they are all orthogonal to $a_{j}$, for all $j \in\{1, \ldots, n\}$.

We define $z_{n+1, k}:=B\left(y_{n+1}, y_{n+1}\right)\left(z_{n, k}\right), k \in N_{n+1}$. Again, Lemma 1.2 assures that $z_{n+1, k}$ is contained in $E \cap E_{0}^{* *}\left(u_{n+1}\right)$. Since $a_{n+1} \in E_{2}^{* *}\left(u_{n+1}\right)$, we deduce that $a_{n+1}$ is orthogonal to each $z_{n+1, k}, \forall k \in N_{n+1}$. Since $y_{n+1}$ and $z_{n, k}$ are orthogonal to $a_{j}$ for all $j \in\{1, \ldots, n\}, k \in N_{n+1}$, using (3), it can be seen that

$z_{n+1, k}=B\left(y_{n+1}, y_{n+1}\right)\left(z_{n, k}\right)=z_{n, k}-2 L\left(y_{n+1}, y_{n+1}\right)\left(z_{n, k}\right)+Q\left(y_{n+1}\right)^{2}\left(z_{n, k}\right)$

is orthogonal to $a_{j}$, for all $j \in\{1, \ldots, n\}, k \in N_{n+1}$. Moreover,

$$
\left\|\sum_{k \in \mathscr{F}} \varepsilon_{k} z_{n+1, k}\right\|=\left\|B\left(y_{n+1}, y_{n+1}\right)\left(\sum_{k \in \mathscr{F}} \varepsilon_{k} z_{n, k}\right)\right\| \leq C,
$$

for any finite subset $\mathscr{F} \subset N_{n+1}$, and $\left|\varepsilon_{k}\right|=1$ in C.

Finally, since $y_{n+1} \in E_{2}^{* *}\left(v_{n+1}\right)$, Lemma 2.1 assures that

$$
\begin{aligned}
\left|\varphi_{k}\left(z_{n+1, k}\right)\right| & \geq\left|\varphi_{k}\left(z_{n, k}\right)\right|-\left|\varphi_{k}\left(z_{n, k}-z_{n+1, k}\right)\right| \\
& >\theta-\frac{336}{\theta} \sum_{j=1}^{n} \delta_{j}-21 \frac{16}{\theta} \delta_{n+1} \\
& =\theta-\frac{336}{\theta} \sum_{j=1}^{n+1} \delta_{j} \quad\left(>\frac{\theta}{2}\right) \quad \text { for all } k \in N_{n+1} .
\end{aligned}
$$

b) $\left.\Rightarrow \mathrm{b}^{\prime}\right)$ Since the elements $\left(a_{n}\right)$ are mutually orthogonal, the subtriple $\mathscr{C}$ generated by the family $\left\{a_{n}: n \in \mathrm{N}\right\}$ coincides with the $\ell_{\infty}$-sum $\bigoplus_{n}^{\infty} E_{a_{n}}$. We recall that each $E_{a_{n}}$ is isomorphic to $C_{0}(L)$, for a suitable locally compact Hausdorff space. Therefore $\mathscr{C}$ is triple-isomorphic to an abelian $\mathrm{C}^{*}$-algebra and the restriction of $K$ to $\mathscr{C}$ cannot be relatively weakly compact.

$\left.\mathrm{b}^{\prime}\right) \Rightarrow \mathrm{a}$ ) is obvious. 
A Dieudonné-type theorem for JC*-triples was established in [11, Theorem 4.2]. When in the proof of the just quoted result, Theorem 2.3 replaces [11, Theorem 3.5], we obtain the following generalization of Dieudonné's theorem in the more general setting of $\mathrm{JB}^{*}$-triples.

TheOREM 2.4. Let $\left(\phi_{n}\right)$ be a sequence in the dual of a JB*-triple $E$ such that the sequence $\left(\phi_{n}(r(x))\right)$ converges whenever $r(x)$ is the range tripotent of a norm-one element $x$ in $E$. Then there exists $\phi$ in $E^{*}$ satisfying that $\left(\phi_{n}\right)$ converges weakly to $\phi$. In particular, if $\left(\phi_{n}(r(x))\right) \rightarrow 0$, for every range tripotent, $r(x)$, of a norm-one element $x$ in $E$, then $\left(\phi_{n}\right)$ is a weakly null sequence in $E^{*}$.

The vector-valued version of the above theorem follows now as a consequence. The following corollary also generalizes the main result in [19] with a shorter and simpler proof.

Corollary 2.5. Let E be a JB*-triple, $X$ a Banach space and $\left(T_{n}\right)$ a sequence of weakly compact operators from $E$ to $X$. Suppose that $\lim T_{n}^{* *}(r(x))$ exists whenever $r(x)$ is the range tripotent of a norm-one element $x$ in $E$. Then there exists a unique weakly compact operator $T: E \rightarrow X$, such that $T^{* *}(z)=\lim T_{n}^{* *}(z)$, for every $z \in E^{* *}$.

Proof. We claim that for each $z \in E^{* *},\left(T_{n}^{* *}(z)\right)$ is a norm convergent sequence. Otherwise, there exist $z \in E^{* *}, \varepsilon>0$, and $(\sigma(n)) \subset \mathrm{N}$ such that $\left\|T_{\sigma(n+1)}^{* *}(z)-T_{\sigma(n)}^{* *}(z)\right\|>\varepsilon, \forall n \in \mathrm{N}$. Defining $S_{k}=T_{\sigma(k+1)}^{* *}-T_{\sigma(k)}^{* *}$, we can find norm-one functionals $\psi_{k} \in X^{*}$ satisfying $\left|\psi_{k}\left(S_{k}(z)\right)\right|>\varepsilon(\forall k \in \mathrm{N})$. Since $T_{k}^{* *}: E^{* *} \rightarrow X^{* *}$ is weak ${ }^{*}$-to-weak* continuous, the sequence $\left(\psi_{k} T_{k}^{* *}\right)_{k \in \mathrm{N}}$ lies, in fact, in $E^{*}$. In particular, the sequence $\left(\psi_{k} S_{k}\right) \subseteq E^{*}$ satisfies, by hypothesis, that $\lim \psi_{k} S_{k}(r)=0$, for every range tripotent, $r=r(x)$, of a norm-one element $x$ in $E$. Theorem 2.4 assures that $\left(\psi_{k} S_{k}\right)$ is weakly null in $E^{*}$, which contradicts $\left.\left|\psi_{k} S_{k}(z)\right|=\mid \psi_{k} S_{k}(z)\right) \mid>\varepsilon,(k \in \mathrm{N})$.

The assignment $z \mapsto L z:=\lim T_{n}^{* *}(z)$ defines a linear mapping $L$ : $E^{* *} \rightarrow X^{* *}$, which is continuous by the Uniform Boundedness Principle. Since each $T_{n}$ is weakly compact we have $T_{n}^{* *}\left(E^{* *}\right) \subseteq X, \forall n \in \mathrm{N}$. In particular $L\left(E^{* *}\right) \subseteq X$. Therefore $T:=L_{\mid E}: E \rightarrow X$ is a well-defined bounded linear operator.

Theorem 2.4 implies that, for each $\psi \in X^{*}$ the $\psi T_{n}^{* *}=T_{n}^{*}(\psi) \in E^{*}$ converge weakly to some $\varphi \in E^{*}$. Thus $\psi L=\varphi \in E^{*}$, which proves that $L$ is weak*-to-weak* continuous. It is now clear that $T^{* *}=L$. Finally, the expression $T^{* *}\left(E^{* *}\right)=L\left(E^{* *}\right) \subseteq X$ shows that $T$ is weakly compact.

ACKNOWLEDGEMENTs. The authors would like to express their gratitude to the anonymous referee whose valuable comments made the presentation more consistent. 


\section{REFERENCES}

1. Akemann, C. A., The dual space of an operator algebra, Trans. Amer. Math. Soc. 126 (1967), 286-302.

2. Barton, T. J., and Friedman, Y., Bounded derivations of JB*-triples, Quart. J. Math. Oxford Ser. (2) 41 (1990), 255-268.

3. Barton, T. J., and Timoney, R. M., Weak ${ }^{*}$-continuity of Jordan triple products and its applications, Math. Scand. 59 (1986), 177-191.

4. Bunce, L. J., Fernández-Polo, F. J., Martínez-Moreno, J., and Peralta, A. M., A Saitô-TomitaLusin Theorem for JB*-triples and applications, Q. J. Math. 57 (2006), 37-48.

5. Burgos, M., Fernández-Polo, F. J., Garcés, J. Martínez, J. and Peralta, A. M., Orthogonality preservers in $C^{*}$-algebras, JB*-algebras and JB*-triples, J. Math. Anal. Appl. 348 (2008), 220-233.

6. Chu, Ch-H., and Mellon, P., JB*-triples have Petczynski's Property V, Manuscripta Math. 93 (1997), 337-347.

7. Diestel, J., Sequences and Series in Banach Spaces, Graduate Texts in Math. 92, Springer, New York 1984.

8. Dineen, S., The second dual of a JB*-triple system, pp. 67-69 in: Complex Analysis, Functional Analysis and Approximation Theory (ed. J. Múgica), North-Holland Math. Studies 125, North-Holland, Amsterdam 1986.

9. Edwards, C. M., review of [11], MathSciNet, Math. Rev. MR2254553 (2007g:46104).

10. Edwards, C. M. and Rüttimann, G. T., Compact tripotents in bi-dual JB*-triples, Math. Proc. Cambridge Philos. Soc. 120 (1996), 155-173.

11. Fernández-Polo, F. J., and Peralta, A. M., Closed tripotents and weak compactness in the dual space of a JB*-triple, J. London Math. Soc. 74 (2006), 75-92.

12. Fernández-Polo, F. J., and Peralta, A. M., A short proof of a Theorem of Pfitzner, Q. J. Math. (to appear).

13. Friedman, Y., and Russo, B., Structure of the predual of a JBW*-triple, J. Reine Angew. Math. 356 (1985), 67-89.

14. Friedman, Y., and Russo, B., The Gelfand-Naimark theorem for JB*-triples, Duke Math. J. 53 (1986), 139-148.

15. Grothendieck, A., Sur les applications linéaires faiblement compactes d'espaces du type $C(K)$, Canadian J. Math. 5 (1953), 129-173.

16. Hanche-Olsen, H. and Størmer, E., Jordan Operator Algebras, Monographs and Studies in Math. 21, Pitman, Boston, MA 1984.

17. Harmand, P., Werner, D. and Werner, W., M-ideals in Banach spaces and Banach algebras, Lecture Notes in Math. 1547, Springer, Berlin 1993.

18. Horn, G., Characterization of the predual and ideal structure of a $J B W^{*}$-triple, Math. Scand. 61 (1987), 117-133.

19. Isidro, J. M., A note on weakly compact operators on $J B^{*}$-triples and a theorem of Dieudonné, preprint 2005.

20. Kaup, W., Algebraic characterization of symmetric complex Banach manifolds, Math. Ann. 228 (1977), 39-64.

21. Kaup, W., A Riemann mapping theorem for bounded symmentric domains in complex Banach spaces, Math. Z. 183 (1983), 503-529.

22. Loos, O., Bounded Symmetric Domains and Jordan Pairs, Math. Lectures, University of California, Irvine 1977.

23. Peralta, A. M., Some remarks on weak compactness in the dual space of a JB*-triple, Tohoku Math. J. 58 (2006), 149-159.

24. Pfitzner, H., Weak compactness in the dual of a $C^{*}$-algebra is determined commutatively, Math. Ann. 298 (1994), 349-371. 
25. Pisier, G., Factorization of Linear Operators and Geometry of Banach Spaces, CBMS Regional Conference Series in Mathematics 60, Amer. Math. Soc., Providence, RI 1986.

26. Takesaki, M., On the conjugate space of operator algebra, Tôhoku Math. J. (2) 10 (1958), 194-203.

27. Takesaki, M., Theory of Operator Algebras I, Springer, New York 1979.

28. Upmeier, H., Symmetric Banach manifolds and Jordan $C^{*}$-algebras, North-Holland Math. Studies 104, Notas de Matemática [Mathematical Notes] 96, North-Holland, Amsterdam 1985.

DEPARTAMENTO DE ANÁLISIS MATEMÁTICO

FACULTAD DE CIENCIAS

UNIVERSIDAD DE GRANADA

18071 GRANADA

SPAIN

E-mail: pacopolo@ugr.es, aperalta@ugr.es 